\title{
Tetraquarks with heavy flavors
}

\author{
D. M. Brink \\ Dipartimento di Fisica, Università degli Studi di Trento, I-38050 Povo (Trento), Italy \\ Fl. Stancu \\ Université de Liège, Institut de Physique B5, Sart Tilman, B-4000 Liège 1, Belgium
}

(Received 4 November 1997; published 12 May 1998)

\begin{abstract}
In the framework of a simple nonrelativistic potential model, we have previously studied the stability of a system of two quarks and two antiquarks of identical light flavor. Here, we extend our analysis to quarks (antiquarks) of different masses and discuss the role of heavy flavors on the stability of the system. This analysis is performed by using a simple variational method which proved powerful in the treatment of other few-body systems. We compare our results with other results from the literature and single out a few characteristics of the spectrum of the tetraquarks. [S0556-2821(98)05811-1]
\end{abstract}

PACS number(s): 12.39.Pn, 12.39.Jh

\section{INTRODUCTION}

The existence of exotic hadrons of the two-quark-twoantiquarks pair type, called tetraquarks or diquonia, is a problem which was already raised about 20 years ago by Jaffe [1] and has been studied within the MIT bag model [1], potential models [2-6], and flux tube models [7]. The MIT bag model indicates the presence of a large number of bound states, the potential models of a few bound states and fluxtube models suggest instability. So far the experimental data have not been conclusive regarding the identification of such states in the light sector, because exotic mesons $q \bar{q} q \bar{q}$ have proved difficult to distinguish from the many conventional $q \bar{q}$ states. But the most recent experiment in this field [8] can revitalize the search. In Ref. [8], it has been shown that the data obtained from the reaction $\pi^{-} p \rightarrow \eta \pi^{-} p$ at $18 \mathrm{GeV} / c$ can be fitted to an exotic meson with $J^{\mathrm{PC}}=1^{-+}$and mass $M=1370 \pm 16 \mathrm{MeV}$. On the other hand, experiments are being planned to search for double-charmed tetraquarks at Fermilab and CERN [9].

From now on, a light quark ( $u, d$, or $s)$ will be denoted by $q$ and a heavy one ( $c$ or $b$ ) by $Q$. It has already been pointed out in the literature that the mass of the quarks play an important role in the stability of a tetraquark system [4-6]. Chiral perturbation theory studies also indicate stability of a tetraquark system with two heavy quarks if $Q$ is the $b$ quark or heavier [10].

In the present work, we focus on the $q q \bar{Q} \bar{Q}$ or, equivalently, the $\bar{q} \bar{q} Q Q$ system, the arguments being given below. Another four-quark system with unequal masses is $q \bar{q} Q \bar{Q}$, which can have two distinct meson-meson thresholds $m_{Q \bar{Q}}$ $+m_{q \bar{q}}$ and $m_{Q \bar{q}}+m_{\bar{Q} q}$. The latter is the same as for $q q \bar{Q} \bar{Q}$, because $m_{\bar{Q} q}=m_{Q \bar{q}}$. It can be shown that [11]

$$
m_{Q \bar{Q}}+m_{q \bar{q}} \leqslant 2 m_{Q \bar{q}},
$$

which means that $Q \bar{Q}+q \bar{q}$ is the lower threshold. Then, assuming that the mass of $q \bar{q} Q \bar{Q}$ is equal to the mass of $q q \bar{Q} \bar{Q}$, the system $q q \bar{Q} \bar{Q}$ has more of a chance to be bound than $q \bar{q} Q \bar{Q}$ and a variational solution, which provides an upper bound, is more conclusive about its stability than for the $q \bar{q} Q \bar{Q}$ system. As an example, detailed arguments in favor of the stability of the ccu $\bar{u}$ system as compared to $c \bar{c} d \bar{u}$ system are given in [9]. Another important difference between $q q \bar{Q} \bar{Q}$ and $q \bar{q} Q \bar{Q}$ systems is that in the latter, there are meson-antimeson annihilation channels, which should also be taken into account [12]. Thus, it is simpler to study $q q \bar{Q} \bar{Q}$ than $q \bar{q} Q \bar{Q}$.

The present work is closely related to that of Zouzou et al. [4] and of Silvestre-Brac and Semay [5,6]. Its purpose is to study $q q \bar{Q} \bar{Q}$ systems with a simple variational method. Our framework is a constituent quark model based on the potential of [13]. This potential generally gives good results both in the meson and the baryon sectors. Moreover, based on the same potential, Silvestre-Brac and Semay made an extensive study of the tetraquarks in a variational calculation with a large oscillator basis for various values of the spin, isospin, and angular momentum. Even in the first step, with 3 or 4 oscillator quanta, these calculations required a basis varying from 10 vectors for a light system to 252 for a heavy one [6].

In the present paper, we explore a different approach based on Gaussian variational wave functions of the type advocated by Kamimura in his studies of nuclear few-body systems [14]. A similar method is widely used in molecular physics. Varga and Suzuki [15] have a recent publication in this area which contains many useful references. A reason for choosing the same potential as Silvestre-Brac and Semay is that we can compare our variational energies with theirs. In the calculations presented below, we show that a variational basis with five simple orbital wave functions is enough to reach convergence for the low-spin and isosopin levels of the $n n \bar{b} \bar{b}$ system, but to values above those given in Refs. [5] and [6]. Thus, some important components are still missing from our wave function. The present paper presents the theoretical framework and some test calculations. It also allows us to draw some conclusions about the spin-isospin 
structure of the spectrum. More work needs to be done to identify the important regions of the variational space.

Zouzou $e$ al. have shown that the $q q \bar{Q} \bar{Q}$ system becomes stable if the mass ratio $m_{Q} / m_{q}$ is large enough. They found that, for values of $m_{Q} / m_{q} \geqslant 20$, only the $\overline{3} 3$ color component of the wave function is important. However, in their paper they give only a schematic discussion of the effect of the spin-spin interaction, while here we consider it explicitly.

In the next section we introduce the Hamiltonian. In Sec. III, we construct the basis states and describe the variational procedure. In Sec. IV, we discuss the role of heavy flavors and, in Sec. V we present numerical results for the $q q \bar{b} \bar{b}$ system. Our study is summarized in the last section.

\section{HAMILTONIAN}

We choose a nonrelativistic Hamiltonian of the form

$$
H=\sum_{i=1}^{4}\left(m_{i}+\frac{\mathbf{p}_{i}^{2}}{2 m_{i}}\right)-\frac{3}{4} \sum_{i<1}^{4} \frac{\lambda_{i}^{c}}{2} \cdot \frac{\lambda_{j}^{c}}{2}\left(V_{i j}^{C}+V_{i j}^{S S}\right),
$$

where $m_{i}$ are the quark masses and $\lambda_{i}^{c}(c=1, \ldots, 8)$ are the $\mathrm{SU}(3)$ Gell-Mann matrices. For the interquark potentials $V_{i j}^{C}$ and $V_{i j}^{S S}$, we choose the following forms [13]:

$$
\begin{gathered}
V_{i j}^{C}=-\frac{\kappa}{r_{i j}}+\frac{r_{i j}}{a_{0}^{2}}-D, \\
V_{i j}^{S S}=\frac{\hbar^{2} c^{2} \kappa}{m_{i} m_{j} c^{4}} \frac{1}{r_{0}^{2} r_{i j}} e^{-r_{i j} / r_{0}} \boldsymbol{\sigma}_{i} \cdot \boldsymbol{\sigma}_{i},
\end{gathered}
$$

where $r_{i j}$ is the distance between the quarks $i$ and $j$ of masses $m_{i}$ and $m_{j}$, respectively. The parameters of Eqs. (2.2) and (2.3) have been chosen to be the same as those used by Silvestre-Brac and Semay $[5,6]$ so that the variational results given in the present paper can be compared with the ones in Refs. [5,6]. The parameter values are given in [13] and were determined, for a choice of the charmed quark mass $m_{c}=1870 \mathrm{MeV}$, by fitting the $1 S, 1 P$, and $2 S$ states of the charmonium. Their values are

$$
\begin{gathered}
\kappa=102.67 \mathrm{MeV} \mathrm{fm}, \quad a_{0}=0.0326\left(\mathrm{MeV}^{-1} \mathrm{fm}\right)^{1 / 2}, \\
D=913.5 \mathrm{MeV}, \quad r_{0}=0.4545 \mathrm{fm} .
\end{gathered}
$$

In [13], the masses $m_{u}=m_{d}=337 \mathrm{MeV}$ were chosen to reproduce the magnetic moments of the nucleon. The bottom quark mass $m_{b}=5259 \mathrm{MeV}$ was obtained by fitting the $Y$ $(1 S)$ meson considered to have a mass of $9434 \mathrm{MeV}$. At the time Ref. [13] appeared, only the $Y(n S)$ mesons $(n=1,2$, 3 , and 4) were experimentally known and the fit seemed to be good for all these mesons. Since then, the experimental spectrum of bottonium has been enriched and improved so that the value of $m_{b}$ found in Ref. [13] underestimates the presently known experimental mass $Y^{\exp }(1 S)=9460 \mathrm{MeV}$. We noticed that there is a difficulty with the parametrization (2.4) to fit precisely both the $Y(1 S)$ and the $B$ meson, unknown at the time this parametrization was produced. Actually, $m_{b}=5259 \mathrm{MeV}$ is a bit too small for reproducing the correct mass of $Y(1 S)$ but too large to fit the $B$ and $B^{*}$

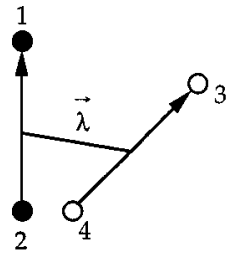

(a)

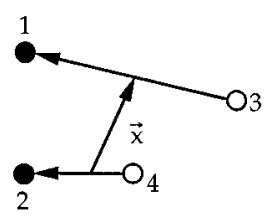

(b)

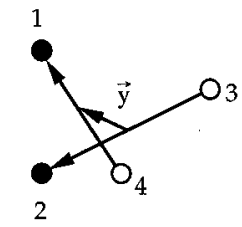

(c)
FIG. 1. Three possible ways to define relative coordinates for a $2 q-2 \bar{q}$ system. Darkened and open circles represent quark and antiquarks, respectively.

meson masses, needed below. However, we are constrained to use the same $m_{b}$ as in Refs. [5,6] in order to compare our variational solution to theirs. Moreover, in the calculated quantity $E-E_{T}$ (see Table IV), $m_{b}$ appears only through Eq. (2.3), so that its fine tuning is not necessary for the present purpose. We chose to study tetraquarks containing $b$ quarks rather than $c$ quarks because with the parameters (2.4) Silvestre-Brac and Semay found that the ground state of the $n n \bar{b} \bar{b}$ is bound, while the ground state of $n n \bar{c} \bar{c}$ is not.

Other choices of the quark Hamiltonian are possible. Recently, the baryon spectroscopy have also been analyzed within a chiral constituent quark model [16] where, instead of one-gluon exchange, the interaction between quarks is due to one-meson (pseudoscalar Goldstone boson) exchange. This makes the spin part of the quark-quark interaction to be flavor dependent. It has been shown $[17,18]$ that the hyperfine splittings and especially the correct order of positive and negative parity states of baryons with $u, d$, and $s$ quarks is better reproduced with this model than with a conventional one-gluon-exchange model, as the one used in this study. Actually, there is evidence from lattice QCD that the hyperfine splittings in light baryons are related mostly to $q \bar{q}$ excitations rather than to forces mediated by gluonic fields in a $q q$ pair [19]. In the present paper, the aim is to explore the variational method based on Gaussian orbital wave functions and we choose to use the Hamiltonian of Ref. [13] for which an elaborate solution $[5,6]$ is available.

In the next section, we introduce internal coordinates for four-body systems and in Sec. IV, we rewrite the Hamiltonian in the internal coordinate system appropriate for the present study.

\section{WAVE FUNCTION}

Here, we present the orbital, color-spin, and flavor structure of a tetraquark system wave function. We suppose that particles 1 and 2 are quarks and 3 and 4 are antiquarks. We choose

$$
m_{1}=m_{2}=m_{q}, \quad m_{3}=m_{4}=m_{Q}, \quad m_{Q} \geqslant m_{q}
$$

\section{A. Orbital part}

One can introduce three alternative coordinate systems. These are (see Fig. 1)

$$
\boldsymbol{\rho}=\frac{1}{\sqrt{2}}\left(\mathbf{r}_{1}-\mathbf{r}_{3}\right), \quad \boldsymbol{\rho}^{\prime}=\frac{1}{\sqrt{2}}\left(\mathbf{r}_{2}-\mathbf{r}_{4}\right),
$$




$$
\begin{gathered}
\mathbf{x}=\frac{1}{2}\left(\mathbf{r}_{1}-\mathbf{r}_{2}+\mathbf{r}_{3}-\mathbf{r}_{4}\right), \\
\boldsymbol{\alpha}=\frac{1}{\sqrt{2}}\left(\mathbf{r}_{1}-\mathbf{r}_{4}\right), \quad \boldsymbol{\alpha}^{\prime}=\frac{1}{\sqrt{2}}\left(\mathbf{r}_{2}-\mathbf{r}_{3}\right), \\
\mathbf{y}=\frac{1}{2}\left(\mathbf{r}_{1}-\mathbf{r}_{2}-\mathbf{r}_{3}+\mathbf{r}_{4}\right), \\
\boldsymbol{\sigma}=\frac{1}{\sqrt{2}}\left(\mathbf{r}_{1}-\mathbf{r}_{2}\right), \quad \boldsymbol{\sigma}^{\prime}=\frac{1}{\sqrt{2}}\left(\mathbf{r}_{3}-\mathbf{r}_{4}\right), \\
\boldsymbol{\lambda}=\frac{1}{2}\left(\mathbf{r}_{1}+\mathbf{r}_{2}-\mathbf{r}_{3}-\mathbf{r}_{4}\right) .
\end{gathered}
$$

In all cases, the fourth coordinate is $\mathbf{R}=(1 / M) \sum m_{i} \mathbf{r}_{i}$ where $M=\Sigma m_{i}$. The coordinates (3.2) or (3.3) are convenient in describing the direct and exchange meson-meson channels, while coordinates (3.4) are handy for incorporating strongly correlated subsystems of identical quarks or antiquarks. A permutation $(i j)$ of two particles $i$ and $j$ applied on the above coordinates gives

$$
\begin{aligned}
& (12) \boldsymbol{\rho}=\boldsymbol{\alpha}^{\prime}, \quad(12) \boldsymbol{\rho}^{\prime}=\boldsymbol{\alpha}, \quad(34) \boldsymbol{\rho}=\boldsymbol{\alpha}, \quad \text { (34) } \boldsymbol{\rho}^{\prime}=\boldsymbol{\alpha}^{\prime}, \\
& \text { (12) } \mathbf{x}=-\mathbf{y}, \quad(34) \mathbf{x}=\mathbf{y}, \quad(23) \mathbf{x}=\boldsymbol{\lambda}, \quad \text { (14) } \mathbf{x}=-\boldsymbol{\lambda}, \\
& \text { (12) } \boldsymbol{\lambda}=\boldsymbol{\lambda}, \quad(34) \boldsymbol{\lambda}=\boldsymbol{\lambda}, \quad(23) \mathbf{y}=\mathbf{y}, \quad \text { (14) } \mathbf{y}=\mathbf{y} .
\end{aligned}
$$

Note that one can also use a coordinate system formed of the vectors $\mathbf{x}, \mathbf{y}, \boldsymbol{\lambda}$ as well. Transformations between various coordinate systems are

$$
\left(\begin{array}{c}
\boldsymbol{\rho} \\
\boldsymbol{\rho}^{\prime} \\
\mathbf{x}
\end{array}\right)=\left(\begin{array}{ccc}
0 & \sqrt{\frac{1}{2}} & \sqrt{\frac{1}{2}} \\
0 & -\sqrt{\frac{1}{2}} & \sqrt{\frac{1}{2}} \\
1 & 0 & 0
\end{array}\right)\left(\begin{array}{l}
\mathbf{x} \\
\mathbf{y} \\
\boldsymbol{\lambda}
\end{array}\right)
$$

$$
=\left(\begin{array}{ccc}
\frac{1}{2} & -\frac{1}{2} & \sqrt{\frac{1}{2}} \\
-\frac{1}{2} & \frac{1}{2} & \sqrt{\frac{1}{2}} \\
\sqrt{\frac{1}{2}} & \sqrt{\frac{1}{2}} & 0
\end{array}\right)\left(\begin{array}{c}
\boldsymbol{\sigma} \\
\boldsymbol{\sigma}^{\prime} \\
\boldsymbol{\lambda}
\end{array}\right),
$$

$$
\begin{aligned}
& \left(\begin{array}{c}
\boldsymbol{\alpha} \\
\boldsymbol{\alpha}^{\prime} \\
\mathbf{y}
\end{array}\right)=\left(\begin{array}{ccc}
\sqrt{\frac{1}{2}} & 0 & \sqrt{\frac{1}{2}} \\
-\sqrt{\frac{1}{2}} & 0 & \sqrt{\frac{1}{2}} \\
0 & 1 & 0
\end{array}\right)\left(\begin{array}{l}
\mathbf{x} \\
\mathbf{y} \\
\boldsymbol{\lambda}
\end{array}\right) \\
& =\left(\begin{array}{ccc}
\frac{1}{2} & \frac{1}{2} & \sqrt{\frac{1}{2}} \\
-\frac{1}{2} & -\frac{1}{2} & \sqrt{\frac{1}{2}} \\
\sqrt{\frac{1}{2}} & -\sqrt{\frac{1}{2}} & 0
\end{array}\right)\left(\begin{array}{c}
\boldsymbol{\sigma} \\
\boldsymbol{\sigma}^{\prime} \\
\boldsymbol{\lambda}
\end{array}\right),
\end{aligned}
$$

$$
\left(\begin{array}{c}
\boldsymbol{\sigma} \\
\boldsymbol{\sigma}^{\prime} \\
\boldsymbol{\lambda}
\end{array}\right)=\left(\begin{array}{ccc}
\sqrt{\frac{1}{2}} & \sqrt{\frac{1}{2}} & 0 \\
\sqrt{\frac{1}{2}} & -\sqrt{\frac{1}{2}} & 0 \\
0 & 0 & 1
\end{array}\right)\left(\begin{array}{l}
\mathbf{x} \\
\mathbf{y} \\
\boldsymbol{\lambda}
\end{array}\right) .
$$

Let us denote by $R^{s}$ the orbital part of the wave function. The most general form with $L=0$ is a function of six scalar quantities expressed in either of the coordinate systems (3.2)-(3.4). We choose the following simple form:

$$
\begin{aligned}
R^{s}= & \exp \left[-\left(A_{11}^{s} \rho^{2}+A_{22}^{s} \rho^{\prime 2}+A_{33}^{s} x^{2}+2 A_{12}^{s} \boldsymbol{\rho} \cdot \boldsymbol{\rho}^{\prime}+2 A_{13}^{s} \boldsymbol{\rho} \cdot \mathbf{x}+2 A_{23}^{s} \boldsymbol{\rho}^{\prime} \cdot \mathbf{x}\right)\right]=\exp \left[-\left(B_{11}^{s} \alpha^{2}+B_{22}^{s} \alpha^{\prime 2}+B_{33}^{s} y^{2}+2 B_{12}^{s} \boldsymbol{\alpha} \cdot \boldsymbol{\alpha}^{\prime}\right.\right. \\
& \left.\left.+2 B_{13}^{s} \boldsymbol{\alpha} \cdot \mathbf{y}+2 B_{23}^{s} \boldsymbol{\alpha}^{\prime} \cdot \mathbf{y}\right)\right]=\exp \left[-\left(C_{11}^{s} \sigma^{2}+C_{22}^{s} \sigma^{\prime 2}+C_{33}^{s} \lambda^{2}+2 C_{12}^{s} \boldsymbol{\sigma} \cdot \boldsymbol{\sigma}^{\prime}+2 C_{13}^{s} \boldsymbol{\sigma} \cdot \boldsymbol{\lambda}+2 C_{23}^{s} \boldsymbol{\sigma}^{\prime} \cdot \boldsymbol{\lambda}\right)\right]
\end{aligned}
$$

These forms of the wave function allow for nonzero angular momenta $l_{12}$ or $l_{34}$ in the subsystems of quarks or antiquarks (see, for example, Tables I, II, or III).

If $R^{s}$ describes an asymptotic channel, it is convenient to use the first or second form of Eq. (3.9) whereas, for a closed channel, the third form is more adequate. The reason is that, using the appropriate coordinate system among Eqs. (3.2)(3.4), one can have a better initial choice of the variational parameters contained in the symmetric matrices $A^{s}, B^{s}$, or $C^{s}$ of Eq. (3.9). But, in the practical calculations of the matrix elements, it may sometimes be convenient to express $R^{s}$ in either form of Eq. (3.9). This can be easily done by knowing the transformation relations between the matrices $A^{s}, B^{s}$, and $C^{s}$ (Appendix A). In the shorthand notation introduced in Appendix A, the wave functions $R^{s}$ can be rewritten as

$$
R^{s}=e^{-X^{T} A^{s} X}=e^{-Y^{T} B^{s} Y}=e^{-L^{T} C^{s} L} .
$$

Our variational solution is a linear combination of the form

$$
\sum_{s} C_{n}^{s} R^{s}
$$


TABLE I. The lowest $q q \bar{Q} \bar{Q} S=0$ states. The color-spin CS states-column 1 -are defined in Eq. (3.18). $C, S$, and $l_{i j}$ stand for color, spin, and orbital angular momenta of the quark or antiquark pairs. $I$ denotes the isospin of the light quark pair, and $P$ denotes the permutation symmetry.

\begin{tabular}{ccccccccc}
\hline \hline & \multicolumn{3}{c}{$\bar{Q} \bar{Q}$} & & \multicolumn{4}{c}{$q q$} \\
CS state & $C^{P}$ & $S^{P}$ & $l_{34}^{P}$ & $C^{P}$ & $S^{P}$ & $l_{12}^{P}$ & $I^{P}$ \\
\hline$\phi_{1}^{0}$ & $3^{-}$ & $1^{+}$ & $0^{+}$ & $\overline{3}^{-}$ & $1^{+}$ & $0^{+}$ & $1^{+}$ \\
$\phi_{2}^{0}$ & $3^{-}$ & $0^{-}$ & $1^{-}$ & $\overline{3}^{-}$ & $0^{-}$ & $0^{+}$ & $0^{-}$ \\
$\phi_{3}^{0}$ & $\overline{6}^{+}$ & $1^{+}$ & $1^{-}$ & $6^{+}$ & $1^{+}$ & $0^{+}$ & $0^{-}$ \\
$\phi_{4}^{0}$ & $\overline{6}^{+}$ & $0^{-}$ & $0^{+}$ & $6^{+}$ & $0^{-}$ & $0^{+}$ & $1^{+}$ \\
\hline \hline
\end{tabular}

where the coefficients $C_{n}^{s}$ are determined by diagonalizing the Hamiltonian (2.1) integrated in the spin-flavor-color space. We are, of course, interested in the lowest state, which corresponds to $n=0$. In the spirit of Refs. [14] and [20], it is important to incorporate in the sum (3.11) all the channels which accelerate the convergence process. This problem will be discussed in Sec. V. Even if the $R^{s}$ belonging to distinct channels are no longer orthogonal, this does not create any problem in the diagonalization procedure. An important advantage of the functions (3.9) is that one can easily perform analytic calculations for a fixed coordinate system, or by choosing a coordinate system in the bra and another in the ket. Our procedure of calculating the overlap $\left\langle R^{s} \mid R^{t}\right\rangle$, the kinetic energy matrix elements $\left\langle R^{s}|T| R^{t}\right\rangle$, and the potential energy matrix elements is given in Appendix B.

\section{B. Spin-color part}

In the color space, one can construct a color singlet $q q \bar{Q} \bar{Q}$ state by using three different couplings, conveniently associated with the three coordinate systems (3.2)-(3.4). The three resulting bases are [1]

TABLE II. Same as Table I, but for $S=1$ and color-spin states defined by Eq. (3.20).

\begin{tabular}{|c|c|c|c|c|c|c|c|}
\hline \multirow[b]{2}{*}{ CS State } & \multicolumn{3}{|c|}{$\bar{Q} \bar{Q}$} & \multicolumn{4}{|c|}{$q q$} \\
\hline & $C^{P}$ & $S^{P}$ & $l_{34}^{P}$ & $C^{P}$ & $S^{P}$ & $l_{12}^{P}$ & $I^{P}$ \\
\hline \multirow[t]{2}{*}{$\phi_{1}^{1}$} & $3^{-}$ & $0^{-}$ & $1^{-}$ & $\overline{3}^{-}$ & $1^{+}$ & $\left\{0^{+}\right.$ & $1^{+}$ \\
\hline & & & & & & $\left\{1^{-}\right.$ & $0^{-}$ \\
\hline \multirow[t]{2}{*}{$\phi_{2}^{1}$} & $3^{-}$ & $1^{+}$ & $0^{+}$ & $\overline{3}^{-}$ & $1^{+}$ & $\int 0^{+}$ & $1^{+}$ \\
\hline & & & & & & $\left\{1^{-}\right.$ & $0^{-}$ \\
\hline \multirow[t]{2}{*}{$\phi_{3}^{1}$} & $3^{-}$ & $1^{+}$ & $0^{+}$ & $\overline{3}^{-}$ & $0^{-}$ & $\int 0^{+}$ & $0^{-}$ \\
\hline & & & & & & $\left\{1^{-}\right.$ & $1^{+}$ \\
\hline \multirow[t]{2}{*}{$\phi_{4}^{1}$} & $\overline{6}^{+}$ & $0^{-}$ & $0^{+}$ & $6^{+}$ & $1^{+}$ & $\int 0^{+}$ & $0^{-}$ \\
\hline & & & & & & $\left\{1^{-}\right.$ & $1^{+}$ \\
\hline \multirow[t]{2}{*}{$\phi_{5}^{1}$} & $\overline{6}^{+}$ & $1^{+}$ & $1^{-}$ & $6^{+}$ & $1^{+}$ & $\int 0^{+}$ & $0^{-}$ \\
\hline & & & & & & $\left\{1^{-}\right.$ & $1^{+}$ \\
\hline \multirow[t]{2}{*}{$\phi_{6}^{1}$} & $\overline{6}^{+}$ & $1^{+}$ & $1^{-}$ & $6^{+}$ & $0^{-}$ & $\int 0^{+}$ & $1^{+}$ \\
\hline & & & & & & $\left\{1^{-}\right.$ & $0^{-}$ \\
\hline
\end{tabular}

TABLE III. Same as Table I, but for $S=2$ and color-spin states defined by Eq. (3.21).

\begin{tabular}{|c|c|c|c|c|c|c|c|}
\hline \multicolumn{5}{|c|}{$\bar{Q} \bar{Q}$} & \multicolumn{3}{|c|}{$q q$} \\
\hline CS state & $C^{P}$ & $S^{P}$ & $l_{34}^{P}$ & $C^{P}$ & $S^{P}$ & $l_{12}^{P}$ & $I^{P}$ \\
\hline \multirow[t]{2}{*}{$\phi_{1}^{2}$} & $3^{-}$ & $1^{+}$ & $0^{+}$ & $\overline{3}^{-}$ & $1^{+}$ & $\int 0^{+}$ & $1^{+}$ \\
\hline & $\bar{\sigma}^{+}$ & $1^{+}$ & $1^{-}$ & $6^{+}$ & $1^{+}$ & $1^{-}$ & $0^{-}$ \\
\hline \multirow{4}{*}{$\phi_{2}^{2}$} & & & & & & $\left\{\begin{array}{l}0 \\
1^{-}\end{array}\right.$ & $1^{+}$ \\
\hline & & \multicolumn{2}{|c|}{$\left|1_{13} 1_{24}\right\rangle}$, & $\left.{ }_{3} 8_{24}\right\rangle$ & & & (3.12) \\
\hline & & \multicolumn{2}{|c|}{$\left|1_{14} 1_{23}\right\rangle$} & $\left.{ }_{4} 8_{23}\right\rangle$ & & & (3.13) \\
\hline & & $\mid \overline{3}_{12} 3$ & & $\left.{ }_{2} \bar{\sigma}_{34}\right\rangle$ & & & (3.14) \\
\hline
\end{tabular}

The first two bases are convenient to asymptotic channels, while Eq. (3.14) is more adequate for closed channels. The 3 and $\overline{3}$ states are antisymmetric and 6 and $\overline{6}$ are symmetric under transposition (12) or (34).

One can use three different couplings in the spin space as well. By coupling four fermions of spin $1 / 2$, the total spin is $S=0,1$, or 2 . For $S=0$, in each coupling, there are two independent basis vectors, as in the color space. The corresponding bases are

$$
\begin{array}{cl}
\left|P_{13} P_{24}\right\rangle, & \left|V_{13} V_{24}\right\rangle, \\
\left|P_{14} P_{23}\right\rangle, & \left|V_{14} V_{23}\right\rangle, \\
\chi_{+}=\left|A_{12} A_{34}\right\rangle, & \chi_{-}=\left|S_{12} S_{34}\right\rangle,
\end{array}
$$

where $A, S, P$, and $V$ stand for axial, scalar, pseudoscalar, and vector mesons subsystems [2]. The definition of each basis vector and the transformations from one basis to another are given explicitly in Appendix A of Ref. [20]. Therefore, for $S=0$, one can introduce the following fourdimensional orthogonal color-spin basis [20]:

TABLE IV. The heavy flavor tetraquark mass $E(q q \bar{b} \bar{b})$ calculated with Gaussian variational wave functions. Column 1: spin $S$, isospin I; column 2: one Gaussian basis; column 3: five Gaussians basis; column 4: results of Refs. [5,6]; column 6: the lowest mesonmeson $M+M^{\prime}$ threshold; column 6: the quantity $E-E_{T}$ where $E_{T}=m_{M}+M_{M^{\prime}}$.

$$
E(q q \bar{b} \bar{b})(\mathrm{MeV})
$$

\begin{tabular}{cccccc}
$S I$ & 1 Gaussian & 5 Gaussians & Brac-Semay & Threshold & $E-E_{T}$ \\
\hline 10 & 10577.7 & 10558.1 & 10525 & $B+B^{*}$ & -98.9 \\
01 & 10802.4 & 10766.2 & & $B+B$ & 156.2 \\
11 & 10812.1 & 10774.1 & 10712 & $B+B^{*}$ & 117.1 \\
21 & 10831.5 & 10789.8 & 10735 & $B^{*}+B^{*}$ & 85.8 \\
\hline \hline
\end{tabular}




$$
\begin{aligned}
& \left|\phi_{1}^{0}\right\rangle=\left|\overline{3}_{12} 3_{34}\right\rangle \chi_{+}, \quad\left|\phi_{2}^{0}\right\rangle=\left|\overline{3}_{12} 3_{34}\right\rangle \chi_{-}, \\
& \left|\phi_{3}^{0}\right\rangle=\left|6_{12} \overline{6}_{34}\right\rangle \chi_{+}, \quad\left|\phi_{4}^{0}\right\rangle=\left|6_{12} \overline{6}_{34}\right\rangle \chi_{-} .
\end{aligned}
$$

In the $S=1$ sector, group-theoretical arguments indicate that there are three spin independent states. We denote these states by

$$
\chi_{1}=\left|V_{12} P_{34}\right\rangle, \quad \chi_{2}=\left|V_{12} V_{34}\right\rangle, \quad \chi_{3}=\left|P_{12} V_{34}\right\rangle
$$

Their definition, properties, and the relation to asymptotic channels are given in Appendix C. Hence, for $S=1$, one deals with a six-dimensional color-spin basis formed of

$$
\left|\phi_{1}^{1}\right\rangle=\left|\overline{3}_{12} 3_{34}\right\rangle \chi_{1}, \quad\left|\phi_{2}^{1}\right\rangle=\left|\overline{3}_{12} 3_{34}\right\rangle \chi_{2}, \quad\left|\phi_{3}^{1}\right\rangle=\left|\overline{3}_{12} 3_{34}\right\rangle \chi_{3}
$$

$\left|\phi_{4}^{1}\right\rangle=\left|6_{12} \overline{6}_{34}\right\rangle \chi_{1}, \quad\left|\phi_{5}^{1}\right\rangle=\left|6_{12} \overline{6}_{34}\right\rangle \chi_{2}, \quad\left|\phi_{6}^{1}\right\rangle=\left|6_{12} \overline{6}_{34}\right\rangle \chi_{3}$.

If the total spin is $S=2$, there is only one spin function which we denote by $\chi^{s}$, see Appendix C. The two color-spin states are

$$
\left|\phi_{2}^{2}\right\rangle=\left|\overline{3}_{12} 3_{34}\right\rangle \chi^{S}, \quad\left|\phi_{2}^{2}\right\rangle=\left|6_{12} \overline{6}_{34}\right\rangle \chi^{S}
$$

\section{Flavor part}

Here, we restrict the discussion to light quarks of type $u$ and $d$. The heavy quarks, $c$ or $b$, have zero isospin, so they do not contribute to the total isospin. Therefore, the total isospin of $q q \bar{Q} \bar{Q}$ can be $I=0$ or 1 . The Pauli principle must be satisfied in each subsystem of identical quarks (antiquarks). Then, only states of internal angular momenta $l_{12}$ and $l_{34}$ of adequate parity are allowed. The possible combinations for the lowest tetraquark states $(l=0$ or 1$)$ with total spin $S=0,1$, and 2 are exhibited in Tables I, II, and III, respectively. In cases where the total orbital angular momentum is $L \neq 0$, the wave functions (3.9) need to be generalized.

\section{LOWEST STATES}

Here, we restrict the color space to the $\overline{3} 3$ component only. The mixing with $6 \overline{6}$ is neglected because one expects it to play a negligible role in deeply bound heavy systems [4], which is the case under consideration here. The appropriate Jacobi coordinate system in such a case is Eq. (3.4) and the spatial part of the wave function $R^{s}$ should be written in the third version of Eq. (3.9).

By removing the center-of-mass motion the kinetic energy takes the form (B4) and (B5) which we denote below by $T_{\mathrm{cm}}$. Then, by integration in the color space, the Hamiltonian (2.1) takes the form

$$
\begin{aligned}
\langle H\rangle_{c}= & \sum m_{i}+T_{\mathrm{cm}}-\frac{3}{4}\left\{-\frac{2}{3}\left(V_{12}+V_{34}\right)\right. \\
& \left.-\frac{1}{3}\left(V_{13}+V_{24}+V_{14}+V_{23}\right)\right\},
\end{aligned}
$$

where

$$
V_{i j}=V_{i j}^{C}+V_{i j}^{S S}
$$

with $V_{i j}^{C}$ and $V_{i j}^{S S}$ defined by Eqs. (2.2) and (2.3). Next, it is useful to integrate in the spin space. The lowest $L=0$ states can be read off Tables I-III. Note that the orbital wave functions have to be symmetric in both $\boldsymbol{\sigma}$ and $\boldsymbol{\sigma}^{\prime}$. Using the spin-spin matrix elements of Appendix C, one finds an expectation value

$$
\langle H\rangle_{\mathrm{cs}}=\sum m_{i}+T_{\mathrm{cm}}+V^{C}+V^{S S}
$$

where

$$
V^{C}=\frac{1}{2}\left(V_{12}^{C}+V_{34}^{C}\right)+\frac{1}{4}\left(V_{13}^{C}+V_{24}^{C}+V_{14}^{C}+V_{23}^{C}\right)
$$

and

$$
V^{S S}= \begin{cases}-\frac{3}{8} V_{12}^{S S}+\frac{1}{8} V_{34}^{S S} ; \quad S=1, \quad I=0 & \\ \frac{1}{8}\left(V_{12}^{S S}+V_{34}^{S S}\right)-\frac{1}{8}\left(V_{13}^{S S}+V_{24}^{S S}+V_{14}^{S S}+V_{23}^{S S}\right) ; \quad S=0, \quad I=1 \\ \frac{1}{8}\left(V_{12}^{S S}+V_{34}^{S S}\right)-\frac{1}{16}\left(V_{13}^{S S}+V_{24}^{S S}+V_{14}^{S S}+V_{23}^{S S}\right) ; \quad S=1, \quad I=1 \\ \frac{1}{8}\left(V_{12}^{S S}+V_{34}^{S S}\right)+\frac{1}{16}\left(V_{13}^{S S}+V_{24}^{S S}+V_{14}^{S S}+V_{23}^{S S}\right) ; & S=2, \quad I=1,\end{cases}
$$


where $V_{i j}^{S S}$ is now given by Eq. (2.3) with $\boldsymbol{\sigma}_{i} \cdot \boldsymbol{\sigma}_{j}$ removed. Before performing practical calculations, it is interesting to consider the limit $m_{Q}$ large, in the spirit of the introductory chapter. Because of the mass dependence exhibited by Eq. (2.3), one expects

$$
V_{12}^{S S} \gg V_{13}^{S S}=V_{24}^{S S}=V_{14}^{S S}=V_{23}^{S S} \gg V_{34}^{S S}
$$

Hence, in this limit, one has

$$
\langle H\rangle_{\mathrm{cs}} \sim\left\langle H_{0}\right\rangle+\left\{\begin{array}{l}
-\frac{3}{8} V_{12}^{S S}, \quad I=0 \\
\frac{1}{8} V_{12}^{S S}, \quad I=1,
\end{array}\right.
$$

where $H_{0}$ stands for all parts of $H$, except for the spin-spin part. Thus, the level with $S=1, I=0$ is expected to be the lowest and the ordering of $I=1$ levels can be found from Eq. (4.5) as $S=0$, the lowest, followed by $S=1$ and $S=2$ on the top. This is indeed the sequence of levels obtained in the studies [5,6] of Silvestre-Brac and Semay when $Q=c$ or $b$ quark. These kinds of remarks can be considered as a guide in practical calculations as presented in the following section or in more elaborate programs, as, for example, that based on stochastic variational methods $[21,22]$.

\section{NUMERICAL RESULTS}

In the following, we wish to test the efficiency of our variational approach applied to the $q q \bar{b} \bar{b}$ with simple trial wave functions. We focus on the lowest $L=0$ states discussed in Sec. IV. In the first step we take a single Gaussian orbital wave function of the form (3.9). For the $\overline{3} 3$ channel, it has to be symmetric in both $\boldsymbol{\sigma}$ and $\boldsymbol{\sigma}^{\prime}$. Hence, $C_{12}=C_{13}$ $=C_{23}=0$. We take $C_{11}, C_{22}, C_{33}$ as variational parameters and minimize the energy of the $S=1, I=0$ state. The minimum occurs for $C_{11}^{0}=C_{33}^{0}=2.9 \mathrm{fm}^{-2}$ and $C_{22}^{0}=18.5 \mathrm{fm}^{-2}$. The corresponding energies $E(q q \bar{b} \bar{b})$ of the multiplet (4.5) are shown in the second column of Table IV.

As a second step we take a more general wave function which is a linear combination of the form (3.11) where all the Gaussians have $C_{12}=C_{23}=C_{31}=0$. Moreover, we choose $C_{11}=C_{33}$ because the minimum for the single Gaussian occurred in this situation. Also, for a single Gaussian, the energy was not very sensitive to $C_{22}$ so we keep this fixed at $C_{22}=18.5 \mathrm{fm}^{-2}$. In the spirit of Kamimura's approach we choose $C_{11}^{n}=\alpha^{n} C_{11}^{0}$ and $C_{22}^{n}=\alpha^{n} C_{22}^{0}$ where $\alpha$ is a parameter. For the results presented in Table IV we take $\alpha=2$. Table IV gives energies calculated with 5 Gaussians $(n=-2$, $-1,0,1,2)$. There is a substantial reduction in the groundstate energy $(\Delta E \sim 21 \mathrm{MeV})$ going from 1 Gaussian to 5 Gaussians. We expect no further significant change in going to more than 5 Gaussians. Values calculated by SilvestreBrac and Semay with a more elaborate variational approach based on a large basis of oscillator wave functions are given in column 4 of Table IV.

In order to discuss the stability of the $q q \bar{b} \bar{b}$ system against strong decays, we also have to calculate the masses of the mesons $M$ and $M^{\prime}$ into which this system can decay. For this purpose, we used the interaction of Sec. II and a two-body wave function of the type $\phi(r)=\sum c_{n} e^{-1 / 2 a_{n}^{2} r^{2}}$ where $r$ is the relative coordinate, $n$ is the number of Gaussians and $a_{n}^{2}$ are in a geometric progression as above. With three Gaussians we found $m_{B}=5304 \mathrm{MeV}$ and $m_{B} * 5352$ $\mathrm{MeV}$, i.e., practically the same values as Silvestre-Brac and Semay. Note that the present experimental values are $m_{B}$ $=5279 \mathrm{MeV}$ and $m_{B *}=5325 \mathrm{MeV}$. In column 6 of Table IV, we indicate the difference between the tetraquark mass $E(q q \bar{b} \bar{b})$ and the threshold mass $E_{T}=m_{M}+m_{M^{\prime}}$. The only bound state in our calculation is the $S=1, I=0$ state, in agreement with Silvestre-Brac and Semay.

Our best variational energy is still about $30 \mathrm{MeV}$ above the ground-state energy obtained by Silvestre-Brac and Semay with the same Hamiltonian. This raises the question about what improvements are still possible within the present approach.

(1) We checked that the results are unsensitive to changes in the value of $\alpha$ in a range 1.5-2.5. Values outside this range were not so satisfactory.

(2) We found that introducing components in the wave function with $C_{11} \neq C_{33}$ produced no significant improvement.

(3) With more Gaussians, one can probably gain another 1 or $2 \mathrm{MeV}$ or binding.

Other possibilities which have not been tried yet are the following:

(4) To include symmetrized components in the orbital state with $C_{12} \neq C_{23} \neq C_{31} \neq 0$.

(5) To incorporate $6 \overline{6}$ channels or alternatively asymptotic channels which are linear combinations of $\overline{3} 3$ and $6 \overline{6}$ channels.

According to Ref. [4], the mixing with $6 \overline{6}$ should be negligible in deeply bound heavy systems. Hence, (4) seems the most favorable possibility.

\section{SUMMARY}

The results presented in Table IV show that the $S=1$, $I=0$ state has a significantly lower energy than the $I=1$ states. Equation (4.5) used in perturbation theory predicts a splitting of the $I=1$ states such that the $(S=0, S=1)$ splitting is one half of the $(S=1, S=2)$ splitting. The variational results with 5 Gaussians show a remarkable agreement with the perturbation result. The present study predicts that the system is bound in the $S=1, I=0$ state and is unbound in the $I=1$ states, in agreement with Silvestre-Brac and Semay.

The expressions given in Sec. III show that the wave functions (3.9) in different channels are related in a simple way through Eqs. (3.2)-(3.4). An advantage of the present approach is that a wave function with a simple angular momentum structure in one channel includes many angular momentum components when transformed to another channel. In the present approach, some basis states can be chosen to include correlations for one of the channels and others for another channel. When one uses an oscillator basis, one chooses from the beginning to work in one of the three basis sets and all the angular momentum components have to be included explicitly.

Our calculations illustrate the merits of the Gaussian 
variational approach in that a substantial lowering of the variational energy was obtained with a relatively simple wave function. On the other hand, our variational energy for the $S=1, I=0$ state is still $33 \mathrm{MeV}$ too high in comparison with the results of Silvestre-Brac and Semay. It remains to be seen if the necessary additional lowering of the energy is due to many small components or if there are one or two important configurations which have not been included so far. A stochastic search of the type advocated by Varga and Suzuki [15] could be useful in locating important regions of the variational space.

\section{APPENDIX A}

In this Appendix, we write explicitly the transformations between the matrices $A^{s}, B^{s}$, and $C^{s}$ introduced in Eq. (3.9). For starting, it is useful to write the coordinates (3.2)-(3.4) in a matrix form as

$$
X=\left(\begin{array}{c}
\boldsymbol{\rho} \\
\boldsymbol{\rho}^{\prime} \\
\mathbf{x}
\end{array}\right) ; \quad Y=\left(\begin{array}{c}
\boldsymbol{\alpha} \\
\boldsymbol{\alpha}^{\prime} \\
\mathbf{y}
\end{array}\right) ; \quad L=\left(\begin{array}{c}
\boldsymbol{\sigma} \\
\boldsymbol{\sigma}^{\prime} \\
\boldsymbol{\lambda}
\end{array}\right)
$$

Then, the exponent of Eq. (3.9), denoted here by $E^{s}$, becomes

$$
E^{s}=X^{T} A^{s} X=Y^{T} B^{s} Y=L^{T} C^{s} L,
$$

where $A^{s}, B^{s}$, and $C^{s}$ are the symmetric matrices introduced in Eq. (3.9), and $X^{T}, Y^{T}$, and $L^{T}$ are the transposes of the matrices (A1).

Introducing the orthogonal matrices

$$
\begin{aligned}
& U_{x}=\left(\begin{array}{ccc}
\frac{1}{2} & -\frac{1}{2} & \sqrt{\frac{1}{2}} \\
-\frac{1}{2} & \frac{1}{2} & \sqrt{\frac{1}{2}} \\
\sqrt{\frac{1}{2}} & \sqrt{\frac{1}{2}} & 0
\end{array}\right) ; \\
& U_{y}=\left(\begin{array}{ccc}
\frac{1}{2} & \frac{1}{2} & \sqrt{\frac{1}{2}} \\
-\frac{1}{2} & -\frac{1}{2} & \sqrt{\frac{1}{2}} \\
\sqrt{\frac{1}{2}} & -\sqrt{\frac{1}{2}} & 0
\end{array}\right),
\end{aligned}
$$

we can write the second transformation of Eqs. (3.6) and (3.7) as

$$
X=U_{x} L ; \quad Y=U_{y} L
$$

and also

$$
Y=U_{y} U_{x}^{-1} X=U_{y x} X .
$$

Using Eqs. (A4) or (A5) in Eq. (A2), one obtains

$$
A^{s}=U_{x} C^{s} U_{x}^{-1} \quad \text { or } C^{s}=U_{x}^{-1} A^{s} U_{x},
$$

$$
\begin{aligned}
& B^{s}=U_{y} C^{s} U_{y}^{-1} \quad \text { or } C^{5}=U_{y}^{-1} B^{s} U_{y}, \\
& B^{s}=U_{y x} A^{s} U_{y x}^{-1} \quad \text { or } A^{s}=U_{y x}^{-1} B^{s} U_{y x},
\end{aligned}
$$

which are the desired transformations. Note that the transformation $A \rightarrow B \rightarrow C$ is equivalent to $A \rightarrow C$ as it should be.

\section{APPENDIX B}

Here, we give the explicit compact form of the matrix elements of the coordinate space part of the Hamiltonian. By $R^{s}$ we understand any of the three alternative forms of Eq. (3.9) so that $A^{s}$ is a generalized notation for the matrix $A^{s}$, $B^{s}$, or $C^{s}$. It is easy to prove that the overlap matrix is

$$
\left\langle R^{s} \mid R^{t}\right\rangle=\left[\frac{\pi^{3}}{\operatorname{det}\left(A^{s}+A^{t}\right)}\right]^{3 / 2} .
$$

Let us generally denote by $\rho$ any of the one-column matrices (A1) and introduce an arbitrary matrix $M$ of the same dimension as $A^{s}, B^{s}$, or $C^{s}(3 \times 3$ in our case $)$. Then, $\rho^{T} M \rho$ is of the same type as the exponent (A2) of Eq. (3.9). Therefore, Eq. (B1) can be generalized to

$$
\begin{aligned}
\left\langle R^{s}\left|e^{-\rho^{T} M \rho}\right| R^{t}\right\rangle & =\left[\frac{\pi^{3}}{\operatorname{det}\left(A^{s}+A^{t}+M\right)}\right]^{3 / 2} \\
& =\left\langle R^{s} \mid R^{t}\right\rangle\left[\frac{1}{\operatorname{det}\left[1+\left(A^{s}+A^{t}\right)^{-1} M\right]}\right]^{3 / 2}
\end{aligned}
$$

Now, suppose that the matrix elements $M_{i j}$ are small. Then, making a Taylor series expansion on both the lefthand side (LHS) and RHS of Eq. (B2) and keeping the linear terms in $M_{i j}$, one obtains

$$
\left\langle R^{s}\left|\rho^{T} M \rho\right| R^{t}\right\rangle=\left\langle R^{s} \mid R^{t}\right\rangle \frac{3}{2} \operatorname{tr}\left[\left(A^{s}+A^{t}\right)^{-1} M\right]
$$

because

$$
[\operatorname{det}(1+F)]^{3 / 2} \cong(1+\operatorname{tr} F)^{3 / 2} \cong 1+\frac{3}{2} \operatorname{tr} F
$$

Expression (B3) can be used in the calculation of the kinetic energy matrix elements. Let us denote by $\boldsymbol{\rho}_{\alpha}$ the elements of any column matrix (A1) corresponding to one of the internal coordinate systems (3.2)-(3.4). Removing the center-of-mass motion, the kinetic energy part of Eq. (2.1) can be generally written as

$$
T=T_{\alpha \beta} \nabla_{\rho_{\alpha}} \cdot \nabla_{\rho_{\beta}}, \quad T_{\alpha \beta}=-\frac{\hbar^{2}}{2 m_{\alpha \beta}} \delta_{\alpha \beta} .
$$

For the particular coordinate system (3.4), the matrix $m_{\alpha \beta}$ is

$$
\begin{gathered}
m_{11}=m, \quad m_{22}=m^{\prime}, \quad m_{33}=\frac{2 m m^{\prime}}{m+m^{\prime}}, \\
m_{12}=m_{23}=m_{31}=0 .
\end{gathered}
$$


Integrating by parts and noting that

$$
\nabla_{\rho_{\beta}} R^{s}=-2 A_{\beta \gamma}^{s} \boldsymbol{\rho}_{\gamma} R^{s}
$$

one obtains

$$
\left\langle R^{s}\left|\nabla_{\rho_{\alpha}} \cdot \nabla_{\rho_{\beta}}\right| R^{t}\right\rangle=-4\left\langle R^{s}\left|A_{\alpha \gamma}^{s} A_{\beta \delta}^{t} \boldsymbol{\rho}_{\gamma} \cdot \rho_{\delta}\right| R^{t}\right\rangle
$$

and

$$
\begin{aligned}
\left\langle R^{s}|T| R^{t}\right\rangle & =-4\left\langle R^{s}\left|\left(A^{s} T A^{t}\right)_{\gamma \delta} \boldsymbol{\rho}_{\gamma} \cdot \boldsymbol{\rho}_{\delta}\right| R^{t}\right\rangle \\
& =-4\left\langle R^{s}\left|\rho^{T} A^{s} T A^{t} \rho\right| R^{t}\right\rangle .
\end{aligned}
$$

Taking

$$
M=A^{s} T A^{t}
$$

in Eq. (B3) leads to

$$
\left\langle R^{s}|T| R^{t}\right\rangle=-6\left\langle R^{s} \mid R^{t}\right\rangle \operatorname{tr}\left[\left(A^{s}+A^{t}\right)^{-1} A^{s} T A^{t}\right],
$$

which is precisely the expression to be used in the calculation of the kinetic energy.

Now, we give some details about the calculation of the potential matrix elements. Let $f\left(\rho_{1}\right)$ be the orbital part of Eqs. (2.2) or (2.3) where $\boldsymbol{\rho}_{1}=\mathbf{r}_{i}-\mathbf{r}_{j}$ for a pair $i j$ of quarks. Choosing the appropriate coordinate system $\rho_{\alpha}$ among Eqs. (3.2)-(3.4), any orbital matrix element can be written in the form

$$
\left\langle R^{s}|f| R^{t}\right\rangle=\int d^{3} \rho_{1} d^{3} \rho_{2} d^{3} \rho_{3} f\left(\rho_{1}\right) \exp \left(-\rho^{T} A^{s t} \rho\right),
$$

where

$$
A^{s t}=A^{s}+A^{t}
$$

In evaluating the integral, the first step is to make a transformation $\boldsymbol{\rho}_{1}, \boldsymbol{\rho}_{2}, \boldsymbol{\rho}_{3} \rightarrow \boldsymbol{\rho}_{1}, \boldsymbol{\rho}_{2}^{\prime}, \boldsymbol{\rho}_{3}^{\prime}$ so that

$$
\rho^{T} A \rho=\left(\boldsymbol{\rho}_{1}, \boldsymbol{\rho}_{2}^{\prime}, \boldsymbol{\rho}_{3}^{\prime}\right)\left(\begin{array}{ccc}
A_{11} & X_{2} & X_{3} \\
X_{2} & \bar{A}_{22} & 0 \\
X_{3} & 0 & \bar{A}_{33}
\end{array}\right)\left(\begin{array}{l}
\boldsymbol{\rho}_{1} \\
\boldsymbol{\rho}_{2}^{\prime} \\
\boldsymbol{\rho}_{3}^{\prime}
\end{array}\right) .
$$

Then, the integrals over $\boldsymbol{\rho}_{2}^{\prime}$ and $\boldsymbol{\rho}_{3}^{\prime}$ can be calculated to give

$$
\begin{aligned}
\left\langle R^{s}|f| R^{t}\right\rangle= & \left(\frac{\pi^{2}}{\bar{A}_{22}^{s t} \bar{A}_{33}^{s t}}\right)^{3 / 2} \\
& \times \int d^{3} \rho_{1} \exp \left(-y^{s t} \rho_{1}^{2}\right) f\left(\rho_{1}\right),
\end{aligned}
$$

where

$$
y^{s t}=A_{11}^{s t}-\frac{X_{2}^{2}}{\bar{A}_{22}^{s t}}-\frac{X_{3}^{2}}{\bar{A}_{33}^{s t}}=\frac{\operatorname{det} A^{s t}}{M_{11}^{s t}}
$$

and

$$
M_{11}^{s t}=\bar{A}_{22}^{s t} \bar{A}_{33}^{s t}=\left|\begin{array}{cc}
A_{22}^{s t} & A_{23}^{s t} \\
A_{23}^{s t} & A_{33}^{s t}
\end{array}\right| .
$$

By integrating over the angles, this reduces to

$$
\begin{aligned}
\left\langle R^{s}|f| R^{t}\right\rangle= & 4 \pi\left(\frac{\pi^{2}}{M_{11}^{s t}}\right)^{3 / 2} \\
& \times \int d \rho_{1} \rho_{1}^{2} f\left(\rho_{1}\right) \exp \left(-y^{s t} \rho_{1}^{2}\right) .
\end{aligned}
$$

The relevant cases for the potentials (2.2) and (2.3) lead to the following integrals:

$$
\text { (a) } f=\mathrm{const} ; \quad \int_{0}^{\infty} d \rho \rho^{2} e^{-y \rho^{2}}=\frac{\pi^{1 / 2}}{4 y^{3 / 2}} \text {, }
$$

(b) $f=r=2^{1 / 2} \rho ; \quad \int_{0}^{\infty} d \rho \rho^{2} r e^{-y \rho^{2}}=\frac{1}{2^{1 / 2} y^{2}}$,

(c) $f=\frac{1}{r}=\frac{1}{2^{1 / 2} \rho} ; \quad \int_{0}^{\infty} d \rho \rho^{2} \frac{1}{r} e^{-y \rho^{2}}=\frac{1}{2^{3 / 2} y^{2}}$,

(d) $\quad f=\frac{1}{r} e^{-r / r_{0}}$;

$\int_{0}^{\infty} d \rho \rho^{2} \frac{1}{r} e^{-r / r_{0}-y \rho^{2}}$

$$
=\frac{1}{2 \sqrt{2} y}\left[1-\left(\frac{\pi}{2 r_{0}^{2} y}\right)^{1 / 2} e^{1 /\left(2 r_{0}^{2} y\right)} \operatorname{erfc}\left(\frac{1}{2 r_{0}^{2} y}\right)^{1 / 2}\right] .
$$

Note that formulas of type (B1), (B6), and (B10) can be easily obtained for the three-particle case and generalized to $n>4$ particles, whenever the Hamiltonian contains a pairwise interaction.

\section{APPENDIX C}

In this Appendix, we give the explicit form of the total spin $S=0,1$ or two wave functions for four-fermion states, where each fermion has $\operatorname{spin} s=1 / 2$ and $s_{z}= \pm 1 / 2$. The notation for spinors is

$$
\left(\begin{array}{l}
1 \\
0
\end{array}\right)=\uparrow, \quad\left(\begin{array}{l}
0 \\
1
\end{array}\right)=\downarrow .
$$

(a) $S=0$ case. The two-basis states $\left|A_{12} A_{34}\right\rangle$ and $\left|S_{12} S_{34}\right\rangle$ of Eq. (3.17) can be defined through their Young tableaux

$$
\chi_{+}=\left|\mathrm{A}_{12} \mathrm{~A}_{34}\right\rangle=\begin{array}{|l|l|}
\hline 1 & 2 \\
\hline 3 & 4 \\
\hline
\end{array}, \quad \chi_{-}=\left|\mathrm{S}_{12} \mathrm{~S}_{34}\right\rangle=\begin{array}{|l|l|}
\hline 1 & 3 \\
\hline 2 & 4 \\
\hline
\end{array}
$$

which exhibit their permutation symmetries

$$
\text { (12) }\left|A_{12} A_{34}\right\rangle=(34)\left|A_{12} A_{34}\right\rangle=\left|A_{12} A_{34}\right\rangle,
$$

$$
\text { (12) }\left|S_{12} S_{34}\right\rangle=(34)\left|S_{12} S_{34}\right\rangle=-\left|S_{12} S_{34}\right\rangle \text {. }
$$

Their explicit form in terms of the spinors (C1) reads [20] 


$$
\begin{gathered}
\chi_{+}=\left|A_{12} A_{34}\right\rangle=\sqrt{\frac{1}{12}}(2 \uparrow \uparrow \downarrow \downarrow+2 \downarrow \downarrow \uparrow \uparrow-\uparrow \downarrow \uparrow \downarrow \\
-\downarrow \uparrow \uparrow \downarrow-\uparrow \downarrow \downarrow \uparrow-\downarrow \uparrow \downarrow \uparrow), \\
\chi_{-}=\left|S_{12}\right\rangle=\frac{1}{2}(\uparrow \downarrow \uparrow \downarrow+\downarrow \uparrow \downarrow \uparrow-\uparrow \downarrow \downarrow \uparrow-\downarrow \uparrow \uparrow \downarrow) .
\end{gathered}
$$

The orthogonal transformations to the other bases are

$$
\begin{gathered}
\left|P_{13} P_{24}\right\rangle=\frac{\sqrt{3}}{2}\left|A_{12} A_{34}\right\rangle+\frac{1}{2}\left|S_{12} S_{34}\right\rangle, \\
\left|V_{13} V_{24}\right\rangle=-\frac{1}{2}\left|A_{12} A_{34}\right\rangle+\frac{\sqrt{3}}{2}\left|S_{12} S_{34}\right\rangle,
\end{gathered}
$$

and

$$
\begin{aligned}
& \left.\left|P_{14} P_{23}\right\rangle=\frac{\sqrt{3}}{2}\left|A_{12} A_{34}\right\rangle-\frac{1}{2}\left|S_{12}\right| S_{34}\right\rangle, \\
& \left|V_{14} V_{23}\right\rangle=-\frac{1}{2}\left|A_{12} A_{34}\right\rangle-\frac{\sqrt{3}}{2}\left|S_{12} S_{34}\right\rangle .
\end{aligned}
$$

(b) $S=1, S_{z}=1$ case. The three independent basis vectors are the three Young-Yamanouchi-type states [23]

$$
\begin{aligned}
& \left|\chi_{1}^{S}\right\rangle=\begin{array}{|l|l|l|}
\hline 1 & 2 & 3 \\
\hline 4 &
\end{array}=\frac{1}{\sqrt{12}}(3 \uparrow \uparrow \uparrow \downarrow-\uparrow \downarrow \uparrow \uparrow-\uparrow \uparrow \downarrow \uparrow-\downarrow \uparrow \uparrow \uparrow \\
& \left|\chi_{2}^{S}\right\rangle=\begin{array}{|l|l|l|}
\hline 1 & 2 & 4 \\
\hline 3 &
\end{array}=\frac{1}{\sqrt{6}}(2 \uparrow \uparrow \downarrow \uparrow-\uparrow \downarrow \uparrow \uparrow-\downarrow \uparrow \uparrow \uparrow) \\
& \left|\chi^{A}\right\rangle=\begin{array}{|l|l|l|}
\hline 1 & 3 & 4 \\
\hline 2 &
\end{array}=\frac{1}{\sqrt{2}}(\uparrow \downarrow \uparrow \uparrow-\downarrow \uparrow \uparrow \uparrow)
\end{aligned}
$$

It is useful to transform this basis into

$$
\begin{gathered}
\chi_{1}=\left|V_{12} P_{34}\right\rangle=\sqrt{\frac{2}{3}} \chi_{1}^{S}-\sqrt{\frac{1}{3}} \chi_{2}^{S}=\frac{1}{\sqrt{2}}(\uparrow \uparrow \uparrow \downarrow-\uparrow \uparrow \downarrow \uparrow), \\
\chi_{2}=\left|V_{12} V_{34}\right\rangle_{1}=-\sqrt{\frac{1}{3}} \chi_{1}^{S}-\sqrt{\frac{2}{3}} \chi_{2}^{S} \\
=\frac{1}{2}(\uparrow \downarrow \uparrow \uparrow+\downarrow \uparrow \uparrow \uparrow-\uparrow \uparrow \uparrow \downarrow-\uparrow \uparrow \downarrow \uparrow), \\
\chi_{3}=\left|P_{12} V_{34}\right\rangle=\left|\chi^{A}\right\rangle=\frac{1}{\sqrt{2}}(\uparrow \downarrow \uparrow \uparrow-\downarrow \uparrow \uparrow \uparrow),
\end{gathered}
$$

where the lower index in |\rangle$_{1}$ stands for $L=1$. For the permutation properties of Eq. (C8), it follows

$$
\begin{aligned}
& \text { (12) } \chi_{1}=\chi_{1}, \quad \text { (12) } \chi_{2}=\chi_{2}, \quad(12) \chi_{3}=-\chi_{3}, \\
& \text { (34) } \chi_{1}=-\chi_{1}, \quad \text { (34) } \chi_{2}=\chi_{2}, \quad \text { (34) } \chi_{3}=\chi_{3} .
\end{aligned}
$$

For the direct asymptotic channel, it is convenient to use the basis

$$
\begin{aligned}
& \left|P_{13} V_{24}\right\rangle=-\frac{1}{\sqrt{2}}\left[\chi_{2}+\frac{1}{\sqrt{2}}\left(\chi_{1}-\chi_{3}\right)\right]=\frac{1}{\sqrt{2}}(\uparrow \uparrow \downarrow \uparrow-\downarrow \uparrow \uparrow \uparrow), \\
& \left|V_{13} P_{24}\right\rangle=-\frac{1}{\sqrt{2}}\left[\chi_{2}-\frac{1}{\sqrt{2}}\left(\chi_{1}-\chi_{3}\right)\right]=\frac{1}{\sqrt{2}}(\uparrow \uparrow \uparrow \downarrow-\uparrow \downarrow \uparrow \uparrow),
\end{aligned}
$$

$$
\begin{aligned}
\left|V_{13} V_{24}\right\rangle_{1} & =-\frac{1}{\sqrt{2}}\left(\chi_{1}+\chi_{3}\right) \\
& =-\frac{1}{2}(\uparrow \uparrow \uparrow \downarrow+\uparrow \downarrow \uparrow \uparrow-\uparrow \uparrow \downarrow \uparrow-\downarrow \uparrow \uparrow \uparrow),
\end{aligned}
$$

and for the exchange channel, the basis

$$
\begin{gathered}
\left|P_{14} V_{23}\right\rangle=-\frac{1}{\sqrt{2}}\left[\chi_{2}-\frac{1}{\sqrt{2}}\left(\chi_{1}+\chi_{3}\right)\right]=\frac{1}{\sqrt{2}}(\uparrow \uparrow \uparrow \downarrow-\downarrow \uparrow \uparrow \uparrow), \\
\left|V_{14} P_{23}\right\rangle=-\frac{1}{\sqrt{2}}\left[\chi_{2}+\frac{1}{\sqrt{2}}\left(\chi_{1}+\chi_{3}\right)\right]=\frac{1}{\sqrt{2}}(\uparrow \uparrow \downarrow \uparrow-\uparrow \downarrow \uparrow \uparrow), \\
(\mathrm{C} 12) \\
\left|V_{14} V_{23}\right\rangle_{1}=\frac{1}{\sqrt{2}}\left(\chi_{1}-\chi_{3}\right) \\
=\frac{1}{2}(\uparrow \uparrow \uparrow \downarrow+\downarrow \uparrow \uparrow \uparrow-\uparrow \uparrow \downarrow \uparrow-\uparrow \downarrow \uparrow \uparrow) .
\end{gathered}
$$

(c) $S=2, S_{z}=2$ case. This is a trivial case, it has a single basis state

$$
\chi^{S}=\uparrow \uparrow \uparrow \uparrow .
$$

The required spin-matrix elements are

$$
\begin{gathered}
\left\langle\chi_{+}\left|\boldsymbol{\sigma}_{1} \cdot \boldsymbol{\sigma}_{2}\right| \chi_{+}\right\rangle=\left\langle\chi_{+}\left|\boldsymbol{\sigma}_{3} \boldsymbol{\sigma}_{4}\right| \chi_{+}\right\rangle=1, \\
\left\langle\chi_{+}\left|\boldsymbol{\sigma}_{1} \cdot \boldsymbol{\sigma}_{3}\right| \chi_{+}\right\rangle=\left\langle\chi_{+}\left|\boldsymbol{\sigma}_{1} \cdot \boldsymbol{\sigma}_{4}\right| \chi_{+}\right\rangle \\
=\left\langle\chi_{+}\left|\boldsymbol{\sigma}_{2} \cdot \boldsymbol{\sigma}_{3}\right| \chi_{+}\right\rangle=\left\langle\chi_{+}\left|\boldsymbol{\sigma}_{2} \cdot \boldsymbol{\sigma}_{4}\right| \chi_{+}\right\rangle=-2, \\
\left\langle\chi_{2}\left|\boldsymbol{\sigma}_{1} \cdot \boldsymbol{\sigma}_{2}\right| \chi_{2}\right\rangle=\left\langle\chi_{2}\left|\boldsymbol{\sigma}_{3} \cdot \boldsymbol{\sigma}_{4}\right| \chi_{2}\right\rangle=1, \\
\left\langle\chi_{2}\left|\boldsymbol{\sigma}_{1} \cdot \boldsymbol{\sigma}_{3}\right| \chi_{2}\right\rangle=\left\langle\chi_{2}\left|\boldsymbol{\sigma}_{1} \cdot \boldsymbol{\sigma}_{4}\right| \chi_{2}\right\rangle \\
=\left\langle\chi_{2}\left|\boldsymbol{\sigma}_{2} \cdot \boldsymbol{\sigma}_{3}\right| \chi_{2}\right\rangle=\left\langle\chi_{2}\left|\boldsymbol{\sigma}_{2} \cdot \boldsymbol{\sigma}_{4}\right| \chi_{2}\right\rangle=-1, \\
\left\langle\chi^{S}\left|\boldsymbol{\sigma}_{i} \cdot \boldsymbol{\sigma}_{j}\right| \chi^{S}\right\rangle=1 \quad \text { for } i<j=1,2, \ldots, 4
\end{gathered}
$$


[1] R.L. Jaffe, Phys. Rev. D 15, 267 (1977); 15, 281 (1977); 17, 1444 (1978).

[2] J. Weinstein and N. Isgur, Phys. Rev. D 27, 588 (1983).

[3] J. Weinstein and N. Isgur, Phys. Rev. D 41, 2236 (1990).

[4] S. Zouzou, B. Silvestre-Brac, C. Gignoux, and J.-M. Richard, Z. Phys. C 30, 457 (1986).

[5] B. Silvestre-Brac and C. Semay, Z. Phys. C 57, 273 (1993).

[6] B. Silvestre-Brac and C. Semay, Z. Phys. C 59, 457 (1993).

[7] G. Dosch, Phys. Rev. D 28, 412 (1983); J. Carlson and V.R. Pandharipande, ibid. 43, 1652 (1991).

[8] D.R. Thompson et al., Phys. Rev. Lett. 79, 1630 (1997).

[9] M.A. Moinester, Z. Phys. A 355, 349 (1996).

[10] A.V. Manohar and M.B. Wise, Nucl. Phys. B399, 17 (1993).

[11] J.-M. Richard, Phys. Rev. A 49, 3573 (1994).

[12] C. Semay and B. Silvestre-Brac, Phys. Rev. D 51, 1258 (1995).

[13] R.K. Bhaduri, L.E. Cohler, and Y. Nogami, Nuovo Cimento A 65, 376 (1981).
[14] M. Kamimura, Phys. Rev. A 38, 621 (1988); Phys. Rev. C 40, 974 (1989).

[15] K. Varga and Y. Suzuki, Phys. Rev. A 53, 1907 (1996).

[16] L.Ya. Glozman and D.O. Riska, Phys. Rep. 268, 263 (1996).

[17] L.Ya. Glozman, Z. Papp, and W. Plessas, Phys. Lett. B 381, 311 (1996).

[18] L.Ya. Glozman, Z. Papp, W. Plessas, K. Varga, and R.F. Wagenbrun, Nucl. Phys. A623, 90c (1997).

[19] K.F. Liu and S.J. Dong, in Proceedings of the 27th International Conference on High Energy Physics, Glasgow, Scotland, 1994, edited by P.J. Bussey and I.G. Knowles (IOP, London, 1995), p. 717, hep-lat/9411067.

[20] D.M. Brink and Fl. Stancu, Phys. Rev. D 49, 4665 (1994).

[21] K. Varga and Y. Suzuki, Comput. Phys. Commun. 106, 157 (1997).

[22] K. Varga, Y. Ohbayasi, and Y. Suzuki, Phys. Lett. B 396, 1 (1997).

[23] Fl. Stancu, Group Theory in Subnuclear Physics (Clarendon, Oxford, 1996), Chap. 4. 